\title{
Meta
}

Journal des traducteurs

Translators' Journal

\section{BASTIN, G. L. and P. F. BANDIA (eds) (2006) : Charting the Future of Translation History, Ottawa, University of Ottawa Press, VII +344 p.}

\section{Serge Marcoux}

Volume 53, numéro 4, décembre 2008

URI : https://id.erudit.org/iderudit/019660ar

DOI : https://doi.org/10.7202/019660ar

Aller au sommaire du numéro

Éditeur(s)

Les Presses de l'Université de Montréal

ISSN

0026-0452 (imprimé)

1492-1421 (numérique)

Découvrir la revue

Citer ce compte rendu

Marcoux, S. (2008). Compte rendu de [BAstin, G. L. and P. F. BANDiA (eds)

(2006) : Charting the Future of Translation History, Ottawa, University of

Ottawa Press, vII + 344 p.] Meta, 53(4), 926-931.

https://doi.org/10.7202/019660ar d'utilisation que vous pouvez consulter en ligne.

https://apropos.erudit.org/fr/usagers/politique-dutilisation/ 
Le Chapitre 9, finalement, contient un résumé des résultats obtenus et des propositions de pistes de recherche qui se dégagent pour l'avenir. On aurait pu espérer ici une évaluation plus systématique des limites apparues avec le deuxième instrument, à savoir Translog. Si ce dernier s'est révélé utile, il présente néanmoins certains inconvénients. Ainsi, le répondant appelé à travailler avec Translog n'aura pas accès aux fonctions avancées offertes par un traitement de texte tel que Word (vérificateur d'orthographe ou encore dictionnaire de synonymes). Il ne pourra pas non plus utiliser des dictionnaires bilingues électroniques intégrés à la plateforme Word. Ces faiblesses n'enlèvent rien à l'importante contribution qu'ont fait les créateurs de Translog à l'étude des processus de traduction. Mais il convient de rappeler que Translog ne donne pas non plus une image tout à fait fidèle des processus cognitifs du traducteur. Son utilisation doit donc faire l'objet d'une décision réfléchie de la part du chercheur. Signalons également, et pour conclure, une piste de recherche très prometteuse proposée par Beate Trandem: l'utilisation d'un outil pour enregistrer les mouvements oculaires des répondants en train de traduire. Cela permettrait en effet d'étudier sur quel segment le traducteur travaille. Cela aiderait aussi le chercheur à comprendre encore mieux l'origine des pauses constatées dans l'exécution d'une tâche de traduction.

En définitive, le travail de Beate Trandem est une contribution réussie au corpus d'études consacrées aux processus de traduction. Il est innovateur en ce sens qu'il combine l'utilisation de différentes méthodes de récolte de données pour étudier un fait langagier spécifique, susceptible d'intéresser des lecteurs qui travaillent dans d'autres couples de langues.

Alexander KüNZli

Université des sciences appliquées de Zurich, Zurich, Suisse

\section{RÉFÉRENCES}

ERICsson, K. A. et Simon, H. A. (1984/1993): Protocol analysis: Verbal reports as data, Cambridge, MIT.

JAKobSEN, A. L. (2003): «Effects of think-aloud on translation speed, revision, and segmentation ", in Alves, F. (ed.), Triangulating Translation: Perspectives in Process Oriented Research, Amsterdam/Philadelphia, John Benjamins, pp. 69-95.

Malblanc, A. (1944): Pour une stylistique comparée du français et de l'allemand, Didier, Paris.

Robertson, S. I. (2001): Problem solving, Hove, Psychology Press.

Vinay, J.-P. and J. Darbelnet (1958): Stylistique comparée du français et de l'anglais, Didier, Paris.

Bastin, G. L. and P. F. Bandia (eds) (2006): Charting the Future of Translation History, Ottawa, University of Ottawa Press, viI +344 p.

Fruit des travaux de la dix-septième conférence annuelle de l'Association canadienne de traductologie, ce recueil de communications vise à établir l'histoire de la traduction comme champ d'étude autonome au sein de la traductologie, que ce soit en redressant certaines erreurs du passé, en comblant certaines lacunes ou en dégageant de nouvelles avenues méthodologiques pour l'avenir.

La première partie du livre aborde ces trois thèmes sous l'angle méthodologique, alors que la deuxième les reprend sous l'angle purement historique.

Julio-César Santoyo, dans sa présentation "Blank Spaces in the History of Translation ", s'intéresse d'abord aux grands oubliés de l'histoire de la traduction. L'histoire de l'interprétation, par exemple, se résume encore trop souvent à une série d’anecdotes illustrant les 
conséquences tragiques d'un choix de mots malheureux. Si la traduction, que ce soit celle des actes juridiques, des textes scientifiques ou des livres religieux, était pratique courante au Moyen Âge, nous ne connaissons presque rien de son évolution chronologique sauf pour la traduction des grands livres, tels la Bible, qui ont marqué leur époque. D’autres histoires restent à écrire: celle des pseudo-traductions ou lettres prétendument rédigées en langue étrangère dans le seul but de permettre à l'auteur de faire preuve d'exotisme; celle des traductions complétées par l'auteur du texte original; celle des traductions dont le texte original est perdu. Viennent ensuite les erreurs historiques qu'il faut redresser, comme cette prétendue «École de Tolède» qui n’a vraisemblablement jamais existé ou la supposée traduction du Coran par Pierre le Vénérable en 1139. Enfin, il y a l'histoire des erreurs tragiques, comme la traduction du mot japonais mokusatsu qui aurait conduit les alliés à larguer les deux premières bombes atomiques sur Hiroshima et Nagasaki.

Paul F. Bandia, pour sa part, étudie «The Impact of Postmodern Discourse on the History of Translation». L'historien de la traduction doit être vu, non comme un traducteur qui «joue» à l'historien, mais comme un véritable historien qui étudie un champ spécifique, celui du discours traduit. Si naguère encore on enseignait qu'il existait une chose telle qu'une correspondance exacte entre théorie et réalité (reconstructionisme) ou à tout le moins une certaine correspondance (constructionisme), l'époque postmoderne affirme que l'histoire est toujours vue à travers le prisme culturel de l'historien (déconstructionisme). À l'époque moderne correspondait une vision du monde basée sur un État-Nation égocentrique; notre époque, celle de la mondialisation, non seulement voit cette notion chanceler, mais considère que toute civilisation est le résultat d'influences diverses venues de mondes extérieurs. De grandes questions comme le postcolonialisme, le féminisme, le rôle des langues mineures en traduction ont détruit le mythe de notre propre infaillibilité. L'historien de la traduction doit savoir utiliser cette analyse et adopter une perspective proactive et interventionniste dans sa propre méthodologie pour jeter les bases d'une discipline résolument moderne, tournée vers l'avenir.

Ce même thème est repris, sur le plan sociologique cette fois, par Reine Meylaerts dans "Conceptualizing the Translator as a Historical Subject in Multilingual Environments». S'appuyant sur l'exemple de son propre pays, la Belgique, elle démontre que lorsque la notion traditionnelle d'État-Nation (un peuple, une langue, un territoire) ne tient plus (dans les États bilingues ou multilingues notamment), la notion d' «habitude» et le processus d'acculturation tendent à rendre perméable, en traduction, la distinction entre langue source et langue cible. Jusqu'en 1935 environ, le français était dans ce pays la langue de la culture, de l'administration et des affaires. Dans la période entre les deux guerres, l'affirmation du flamand a valu à de nombreuses œuvres d'être traduites de cette langue vers le français. Tout en démontrant l'importance grandissante de cette langue, elle confirmait en même temps son statut d'infériorité puisque les francophones, au lieu d'apprendre le flamand pour pouvoir lire les textes en langue originelle, les faisaient traduire dans leur langue. Si bien qu'un traducteur comme Roger Kervyn, pourtant francophone, qui avait réussi à créer une langue littéraire reproduisant le parler "petit flamand» du quartier des Marolles à Bruxelles, sentant l'inconfort de sa position, décida de mettre un terme à son œuvre alors au sommet de sa popularité. Une telle situation ne pourrait se produire au Canada où les processus d'acculturation historique, culturelle et politique ont évolué d'une tout autre façon. D’où la nécessité de repenser les notions de «culture source» et de «culture cible» et la position du traducteur dans le processus de communication entre les deux.

Le courant de recherche «microhistorique» propose de délaisser les phénomènes de groupes pour se concentrer sur l'individu et de s'en inspirer pour voir comment celui-ci reflète le monde qui l'entoure. Sergia Adamo revoit ainsi l'histoire de la traduction à la lumière de la microhistoire. Dans «Microhistory of Translation », elle résume ce mouvement en mettant en lumière des sources et documents d'archives jusque là délaissés, ce qui permet 
de repenser à la fois la notion narrative de l'histoire et la position subjective de l'historien puisque les gestes que fait celui-ci en choisissant ses champs de recherche ne sont pas neutres et trahissent une réinvention de l'histoire pour lui donner un sens face au présent. Ses recherches lui ont ainsi permis d'établir que, dans l'espace culturel italien, la traduction de romans au dix-huitième siècle s'avérait un facteur important pour identifier un public lecteur féminin en tant que marché ayant ses caractéristiques propres.

En ce qui a trait à l'interprétariat, on pourrait avancer que l'histoire de cette discipline n'existe pratiquement encore qu'à l'état de microhistoire. S'inspirant du cas de Joseph Belleau, un avocat de Québec qui, en 1919, fut recruté pour servir d'interprète à la conférence internationale qui devait donner naissance à l'Organisation mondiale du travail, Jésus Baigorri Jalón, dans «History of Interpretation», montre le travail qu'il reste à faire pour passer du cas d'espèce à la rédaction d'une histoire de l'interprétation. Pour y parvenir, il trace un tableau fascinant de dix-sept champs d'études possibles, en identifiant les sources disponibles pour chacun, les méthodes de travail à employer, et les difficultés que l'on doit s'attendre à y rencontrer.

Si les premières contributions s'attachaient surtout à combler certaines lacunes que contient encore l'histoire de la traduction, nous commençons avec «Subjectivity and Rigour in Translation History» de Georges L. Bastin, la rectification de diverses erreurs qui ont toujours cours. Ainsi notre vision eurocentrique de l'histoire a longtemps conduit, dans le cas de l'Amérique latine, à adopter une attitude paternaliste, voire raciste, à l'endroit des cultures de ce continent, attitude qui a longtemps empêché de saisir divers concepts locaux indispensables à la compréhension de sa complexité culturelle faite de contrastes et d'oppositions. L'auteur en tire comme conséquence que la rigueur de l'historien doit le conduire d'abord à refuser les «modèles importés» qui ne s'adaptent pas à la réalité et à construire ensuite de nouveaux modèles qui, pour subjectifs qu'ils soient, incorporeront des sources primaires jusque là négligées comme les pamphlets, les chansons populaires, voire les graffitis.

Rectification historique également, l'exposé de Clara Foz intitulé «Translation, History and the Translation Scholar», portant sur deux notions: le rôle de l'histoire et la périodisation. Soulignant la contribution d'un site web comme «Historia a debate ${ }^{1}$ », l'auteure rappelle les grands débats ayant entouré l'existence éventuelle d'une "vérité historique», tout en soulignant que la multitude d'interrogations sur le sujet permet au moins d'avoir conscience du problème et d'éviter de tomber dans les pièges tendus sous les pieds des historiens des siècles passés, que ce soit dans l'histoire en général ou dans l'histoire de la traduction. Quant à la périodisation, visant à délimiter des "façons de traduire» au cours des siècles sur le modèle des grandes périodes de l'histoire, on sait à quel point elle est à la fois nécessaire et arbitraire. La pratique de la traduction tout comme la réflexion sur celle-ci sont donc basées sur des constructions établies par différents spécialistes en fonction de leurs intérêts et de leur temps; elles doivent dès lors être analysées et corrigées si besoin est.

La deuxième partie de l'ouvrage réunit des exposés qui reprennent ces questions méthodologiques mais en portant l'accent sur des champs d'étude particuliers (ClaireHélène Lavigne sur l'histoire de la traduction juridique), des régions géographiques (Christine York sur la vision que se faisaient les Européens du Nouveau Monde) ou des périodes historiques (Chantal Gagnon sur l'histoire de la traduction des discours politiques au Canada).

D’emblée, Claire-Hélène Lavigne annonce les couleurs en donnant comme sous-titre à son exposé «Literalness and Legal Translation»: «Myth and False Premises». On a longtemps prétendu que, si les textes juridiques avaient été traduits de façon très littérale jusqu'au début du dix-septième siècle, c'était parce que, à l'instar de la Bible, ils faisaient autorité en leur domaine, étant en quelque sorte la loi de Dieu édictée par le Prince. Au contraire, prenant comme exemple le Corpus Iuris Civilis rédigé au sixième siècle (le Code justinien), 
l'auteure démontre dans la traduction qu'en fait Richard d'Annebaut pour l'édification de son pupille, Bertrand d'Escalphepié, en 1280, comment la façon de traduire est étroitement liée au but du traducteur, à la période de l'histoire où le texte a été traduit et à la culture juridique cible.

Marilyn Gaddis Rose s'attaque à un domaine bien particulier, celui du roman historique et de ses traductions dans "The Role of Translation in History, The Case of Malraux". Utilisant comme point de départ les traductions en langue anglaise de Les Conquérants (1928), traduit en 1929 par Winifred Stephens Whale et en 1976 par Stephen Becker, ainsi que La Condition humaine (1933), traduit l'année suivante par Haakon M. Chevalier et en 1934 par Alastair MacDonald, elle met en relief les connotations colonialistes des textes de Malraux décrivant la vie à Saïgon et à Canton et montre comment les traducteurs ont rendu celles-ci dans leurs traductions par la description suffisante et condescendante des natives, réduits au niveau d'objets, plutôt que comme sujets de ces deux romans. Avec le recul du temps, on peut comprendre comment la lecture préexistentialiste qui en était faite dans les années 1930 a cédé la place à l'agacement postcolonial des années d'après-guerre.

L'histoire de la traduction en langue hébraïque est présentée dans l'Encyclopédie hébraïque de Toury (1980) comme une partie intégrante du renouveau de la langue, fossilisée après des siècles de strict respect de la littérature canonique. Dans son article «Puritan Translations in Israel, Rewriting a History of Translation", Nitsa Ben-Ari remet en question cette notion en montrant comment en fait une forme de puritanisme victorien lié à l'idéologie sioniste a conduit à une censure de l'érotisme se dégageant des textes originaux, laquelle a eu ses répercussions sur l'évolution de la langue. Si la torah n'était pas exempte d'allusions sexuelles, l'image du Nouveau Juif, celui qui avait survécu aux ghettos et qui avait fondé Israël, se devait de souligner le caractère «viril» de la nouvelle nation et de ses fondateurs. Les traductions auraient pu en principe échapper à cette autocensure puisqu'elles reflétaient simplement des phénomènes se produisant dans d'autres cultures. Pourtant que ce soit sur le plan de la littérature salace ou dans les textes pseudo-médicaux, ou même dans les traductions de romans étrangers comme Tropique du Cancer de Henry Miller, traduit trente ans après sa parution, on note soit la suppression de passages érotiques, soit le remplacement de termes «offensants» par des euphémismes ou des adaptations de termes allemands qui non seulement en rendaient la lecture difficile, mais enrayaient le développement du vocabulaire propre au genre.

Cette influence de l'idéologie politique dans la traduction n'est pas le seul fait d'Israël. Le Canada bilingue en présente également des exemples comme le montre Chantal Gagnon dans son étude «Ideologies in the History of Translation, A Case Study of Canadian Political Speeches». L'histoire des deux principales communautés repose sur des ensembles de valeurs différentes que les discours politiques, visant à maintenir l'unité du pays, se devaient de ne pas exacerber surtout en temps de crise. S'appuyant sur le modèle théorique du Critical Discourse Analysis et de la taxonomie de Chesterman, l'auteure analyse un corpus de sept discours politiques prononcés entre 1942 et 1995, pour établir comment les variations notées dans les traductions reflétaient des notions distinctes d'identité nationale et correspondaient (1) au contexte historique dans lequel était prononcé le discours, (2) à l'endroit où il était prononcé, (3) à l'auditoire devant lequel se trouvait le premier ministre et (4) à l'appartenance ethnique de ce dernier.

Remontant plus loin dans l'histoire du Canada, Jo-Anne Elder explique dans « Keepers of the Stories", que le traducteur n'est pas seulement le gardien de l'Histoire mais qu'il peut également la créer. En effet, certaines langues peuvent disparaître et avec elles les histoires et les légendes qu'elles véhiculaient. Celui qui nous transmet ces légendes en les rendant accessibles dans notre langue les préserve ainsi de l'oubli et se fait créateur de l'Histoire, comme c'est le cas de Robert Bringhurst, poète et traducteur, qui s'est attaché aux mythes des Haïda Gwaii. Créateur et écrivain, Bringhurst l'est déjà par le fait qu'il met par écrit des 
récits à l'origine exclusivement oraux. Anthropologue et traducteur, plutôt que de faire passer le message des anciens Haïda dans notre langue et notre culture, il tente de nous amener à comprendre la culture haïda en respectant d'abord le nom des individus : tout en utilisant la langue des colonisateurs, il préfère parler de Daxhiigang plutôt que d'Edenshaw, de Kilxhawgins plutôt que d'Abraham Jones, nous forçant ainsi à entrer dans cette culture. De plus, ses textes maintiennent la syntaxe et les noms composés originaux, quitte à utiliser de nombreuses notes de bas de page comme, par exemple: "Then ${ }^{\star}$ thus ${ }^{\star}$ him ${ }^{\star}$ he ${ }^{\star}$ talkingacting ${ }^{\star}$ him-to ${ }^{\star}$ he ${ }^{\star}$ grasping-handling-the ${ }^{*}$ when/Me ${ }^{\star}$ here ${ }^{*}$ are. Nul doute que ce genre de texte ne paraisse rebutant au premier abord, mais c'est par ce truchement que nous pouvons entrer dans l'esprit d'une civilisation menacée de disparition.

Suit un article qui interrompt la suite des textes consacrés à l'Amérique. Ernest Bramah Smith acquit la célébrité au tout début du $\mathrm{xx}^{\mathrm{e}}$ siècle grâce à ses pseudo-traductions. Dans "Long Time No See, Coolie», James St. André analyse les procédés par lesquels cet auteur, en s'inspirant des nombreuses traductions qui avaient été à la mode au siècle précédent, a su créer un style reproduisant les caractéristiques de ce que l'on considérait comme typiquement chinois, à savoir l'abondance de proverbes et de maximes, une politesse frôlant l'obséquiosité, un langage rempli d'images et une pléthore d'adjectifs et d'adverbes. En même temps, toutefois, son œuvre a contribué à perpétuer, voire à répandre l'image à la fois condescendante et colonialiste que se faisaient les Britanniques de l'empire du Milieu. Il est donc nécessaire selon St. André de reprendre toute l'histoire de la traduction du chinois vers l'anglais à partir de l'époque des premiers missionnaires jusqu'à nos jours pour y distinguer la réalité historique du pastiche et en tracer un nouveau portrait plus conforme celui-là à la réalité historique.

Lourdes Arencibia Rodriguez nous ramène en Amérique, plus précisément dans la première école de traducteurs et interprètes du seizième siècle, "The Imperial College of Santa Cruz de Tlatelolco». Perdu pendant près de trois siècles, l'immense travail de compilation et de traduction accompli dans ce collège impérial, où étaient éduqués les fils de la noblesse indigène mexicaine, a été retrouvé depuis peu. Il permet de prendre conscience des énormes difficultés à la fois culturelles et linguistiques auxquelles furent confrontés et les premiers enseignants européens et leurs élèves autochtones pour réconcilier l'humanisme européen tel qu'il résultait du concile de Trente (1564) et la conception du monde que se faisaient les Mexicains. En fait, l'éradication de l'idolâtrie, de ses temples, monuments et écrits par les premiers missionnaires avait si bien réussi que ce n’est que par les souvenirs transmis au cours de longues veillées par les anciens qu'il s'avérait possible de faire passer les concepts européens dans la mentalité mexicaine et, ensuite, de les traduire dans les langues locales, avec toutes les variations que cela pouvait représenter tant sur le plan des sources premières que des reproductions qui en furent faites par la suite.

À ces difficultés s'ajoutaient, en Amérique du Sud, les politiques linguistiques toujours changeantes du colonisateur, l'apparition de dialectes espagnols dans les Andes et l'impossibilité de rendre le dogme catholique dans les langues indigènes. C'est ce que nous explique Lydia Fossa dans sa description du projet Glosas croniquenses: A Synchronic Bilingual (American Indigenous Languages - Spanish) Set of Glossaries. Ces chroniques visent essentiellement à identifier et à récupérer le patrimoine lexical et sémantique des langues indigènes en donnant priorité à celles des Andes (quechua, aimara, puquina, taino, kuná et náhuatl). En même temps, elles cherchent à établir les équivalents espagnols de ces termes au seizième siècle. L'auteure nous explique comment furent élaborées et mises en œuvre les différentes étapes de ce projet, dont deux sont pratiquement complétées, tout en invitant les chercheurs à consulter les trois glossaires déjà disponibles sur la Toile.

L'un des buts que peut se donner la relecture des récits de voyage et de découverte du seizième siècle est d'identifier les voix du passé qui ne reflètent pas le projet et l'idéologie du colonisateur. L'une de ces voix, que nous présente Christine York dans sa contribution 
«Translating the New World» est celle de Jean de Léry et de son Histoire d'un voyage fait en la terre du Brésil. De Léry était un calviniste français qui, à la fin du seizième siècle, alors que la France cherchait à s'établir en Amérique du Sud, conduisit une expédition qui lui fit rencontrer les Indiens Tupinamba du Brésil chez qui il demeura plusieurs mois. S’intéressant il est vrai plutôt à la faune et à la flore qu'à la conversion des indigènes, de Léry utilise surtout dans ses textes les termes tupinamba au lieu de chercher à leur donner des équivalents français, ne les «traduisant» qu'en donnant une forme écrite à des mots appartenant jusque là à une civilisation orale. En utilisant la taxonomie en usage au seizième siècle, ellemême basée sur celle de Pline, il aura ainsi contribué à «nommer» le Nouveau Monde puisque c'est sur la base de ce vocabulaire que nous parlons encore de nos jours d' "ananas", de «manioc», de "caïman» et de noix d' "acajou».

Avec le dernier texte, nous retournons en Espagne où un jeune voyageur britannique, Robert Southey (1774-1843), invité par son oncle, pasteur de la colonie britannique de Lisbonne, se prit de passion pour l'histoire de l'Espagne et du Portugal, deux pays dont il maîtrisa rapidement les langues. Dans sa contribution, "Amadis of Gaul (1803) and Chronicle of the Cid (1808) by Robert Southey», Juan Miguel Zarandona s'intéresse surtout au facteur humain en traduction et à l'histoire de la traduction vue par les yeux des traducteurs eux-mêmes. À travers ces deux traductions d'épopées historiques, il explique comment Southey est resté fidèle aux principes qu'il avait formulés: prendre des libertés plutôt que de risquer de lasser le lecteur par un mot à mot trop strict; compresser à condition de ne pas perdre ce qui fait la richesse de l'original; respecter la balance des éléments constituant le texte original; conserver le style de l'époque là où c'est possible; porter une grande attention à la traduction des noms propres (une façon de maintenir l'exotisme du texte); faire de la modestie la vertu primordiale du traducteur. Particulièrement intéressant est le fait que la Chronique du Cid n'est pas la traduction d'un seul texte, mais bien l'amalgame d'une chronique, d'un long poème et de ballades dont Southey retint, dans sa traduction, les éléments les plus intéressants.

Selon le vœu des compilateurs, il est à souhaiter que ces exposés sur la méthodologie et l'histoire de la traduction donnent une nouvelle image de l'histoire de la traduction en tant que discipline autonome et ouvrent la voie à de nouvelles recherches dans un secteur où beaucoup reste à faire.

Serge Marcoux

Université de Montréal, Montréal, Canada

\section{NOTE}

1. <http://www.ensayistas.org/critica/manifiestos/H-debate.htm>. La version originale est en espagnol; il existe toutefois une version anglaise et française.

Folkart, B. (2007): Second Finding: A Poetics of Translation, Ottawa, University of Ottawa Press, 562 p.

There are nine chapters in this book, essays on translation, each one with a particular slant on the complexity of "unpacking" the truth of the literary text and the challenges of translating it.

Multiple insights into literary translation are here, translation of poetry in particular. Some of the chapters are good résumés of theory and practice already known to the specialist but the section that appeals to me most is "Poetry as Knowing," a brilliant analysis of the poem as an "inexhaustible inscape" and the fact that translation can lift the veils of a poem in an unrelenting effort to remove the last veil and reveal the truth of the text. The latter will never be the same as the original, obviously not in terms of signs, but usually not 